\begin{tabular}{ccc}
\hline & International Journal of Engineering \& Technology, $7(2.12)(2018) 8-10$ \\
SPC & International Journal of Engineering \& Technology \\
Website: $w w w . s c i e n c e p u b c o . c o m / i n d e x . p h p / I J E T$ & Research Paper \\
\hline
\end{tabular}

\title{
Degradation evaluation of high voltage insulating oils by terahertz spectroscopy
}

\author{
Ki-Chul Kim * \\ Department of Advanced Chemical Engineering, Mokwon University, 88 Doanbook-ro, Seo-gu, Daejeon, 35349, Republic of KOREA \\ *Corresponding author E-mail:
}

\begin{abstract}
Background/Objectives: The stable electrical power supply is the most important issue for modern industrial society employing various electrical appliance. The power transformer is one of the most important component in the electrical power supply system.

Methods/Statistical analysis: The common diagnosis method of insulating oil of transformer is gas-chromatograph. The gas-chromatograph is performed to detect dissolved gas ininsulating oil of transformerduring the stop-period of transformer unit. In this research, the optical properties of insulating oils of transformer (unused new oil, used oil of normal operation, and waste oil of malfunction) are analyzed using a terahertz time-domain spectroscopy in the range of $0.1 \sim 3.0 \mathrm{THz}$.

Findings: The new insulating oil is colorless and transparent. The used insulating oil and waste insulating oil exhibit dark yellow and brown color. The THz sample pulses are delayed of phase and reduced of amplitude compare to passing through the free space. The reduce of amplitude is attributed to increasing of absorption due to aging effect of oils. The refractive index and absorption coefficient of insulating oils are calculated from measured THz pulses. The refractive index of 'new or used oil' exhibits a constant value of 1.455 and 1.463 in the full frequency range. But refractive index of 'waste oil' exhibits various values of $1.466 \sim 1.485$ with the frequency, which is relatively high. The normalized amplitude of waste insulating oil is reduced. The absorption coefficient of waste insulating oil is increased linearly with the increase of frequency. The reducing of $\mathrm{THz}$ amplitude is attributed to increase of absorption due to aging effect of oils.

Improvements/Applications: The aging effect of insulating oils of transformer is characterized by the portable THz-TDS system. It can be applied to in situ monitoring of insulating oil conditions.
\end{abstract}

Keywords: Terahertz Spectroscopy; Insulating Oil; Refractive Index; Dielectric Constant; Absorption Coefficient; Degradation.

\section{Introduction}

The modern industrial society has been used various electrical appliance such as refrigerator, air conditioner, TV, computer etc. The stable electrical power supply is the most important issue for modern industrial society. The power transformer isone of the most important and cost-intensive component in the electrical power supply system. An unexpected outage results in substantial costs mainly caused by the outage of the power substation ${ }^{1}$. Therefore, a big interest and various methods for diagnosis of power transformer conditions are given [2-5]. The most common insulation system for power transformer is oil-board insulating system. Diagnostic methods of power transformer are broadly categorized such as chemical diagnostic methods, electrical diagnostic methods, optical/thermal diagnostic methods, and mechanical diagnostic methods. The most effective characterization method of insulating oil is the gas-chromatograph, thechemical methods which has high sensitive sensors, to detect internal gas (dissolved gas, gas-in-oil) of transformer. The maintain of electrical power quality is controlled by time based maintenance system. The electrical substations are equipped the parallel electrical power transformer units under time based maintenance system. The diagnosis of insulating oils of power transformer is performed by gas-chromatograph analysis in stop-period of power transformer unit [6-7]. The development of real time monitoring technique of insulating oil conditions is requested for effective condition based power maintenance system. Real time monitoring of insulating oil characteristics is possible with using an UV- visible spectroscopy, FT-IR spectroscopy, and Terahertz (THz) spectroscopy [8-10].

Terahertz wave is the part of electromagnetic spectrum between the microwave and far infrared bands ( 0.1 to $10 \mathrm{THz}$ ). THz-TDS (timedomain spectroscopy) has been employed to investigate a wide variety of materials, including liquids [11], graphene [12], paints [1314], ceramics [15], and biomolecules [16].

In this paper, degradation evaluation of insulating oils of high voltage power transformer is characterized by THz-TDS for aging effect of insulating oils. The optical properties of insulating oils, such as the refractive index, absorption coefficient, dielectric constant, and tan delta are analyzed in the spectral range of 0.1 to $3.0 \mathrm{THz}$.

\section{Experimental details}

The THz-TDS system has conventional transmission geometry. In general, THz-TDS systems are consisted with bulk optical components which have beam alignment problems. But developed our portable THz-TDS system is beam alignment issues free due to the employing of single mode optical fibers. Optical pulses with pulse width of approximately $90 \mathrm{fs}$ at $1560 \mathrm{~nm}$ are delivered from a modelocked optical fiber laser at a repetition rate of $100 \mathrm{MHz}$. To generate $\mathrm{THz}$ pulse, the pumped beam is focused on the biased gap of a photoconductive antenna fabricated on a low-temperature-grown GaAs (LT-GaAs) layer. The THz pulse is measured in a phase sensitive detection circuit by lock-in amplifier (Stanford Research System, SR830). The THz waves are seriously affected by water vapor, 
thus the system is enclosed in a chamber which is purged with dry air to reduce $\mathrm{THz}$ absorption due to residual water vapor in the $\mathrm{THz}$ beam path. The measurements are carried out at room temperature and $1 \%$ of relative humidity.
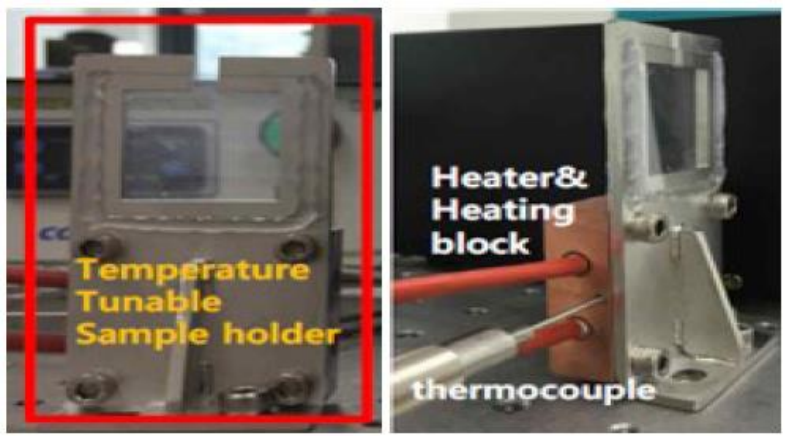

Fig. 1: Digital Photos of Thz Liquid Sample Holder and Liquidsample Cell

The liquid sample holder is fabricated with the aluminum plate and polypropylene (PP) plates which are $\mathrm{THz}$ window, and has $2 \mathrm{~mm}$ thickness as shown in Figure 1. The sample cell shape is a rectangular slab with $5 \mathrm{~mm}$ interspace thickness. The sample holder is assembled on heating block of copper.The heater and thermocouple are embedded into heating block. The heating block is controlled with $1^{\circ} \mathrm{C}$ accuracy.The Korean Electric Power Corporation (KEPCO) is offered insulating oils to analyze the aging effect of insulating oils. The 'waste insulating oil' was extracted from the malfunction transformer. The 'used insulating oil' was extracted from the normal operation transformer. The last 'new insulating oil' is a fresh insulating oil which is an unused oil. The KEPCO has several kinds of insulating oils. But the offered insulating oils are same types in this analysis. Figure 2 is a digital photograph of insulating oils. The new insulating oil is colorless and transparent. The used insulating oil and waste insulating oil exhibitdark yellow color and brown color.

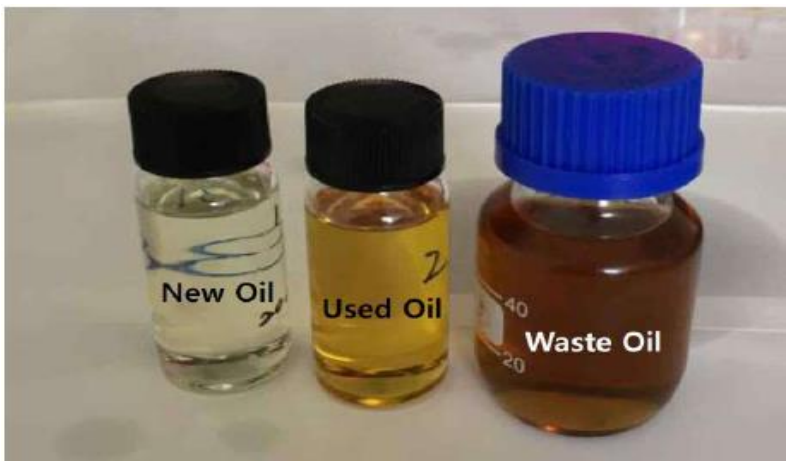

Fig. 2: Digital Photos of Insulating Oils which are Characterized by ThzTDS (Unused New Oil, Used Oil of Normal Operation, and Waste Oil of Malfunction).

\section{Results and discussion}

The measured $\mathrm{THz}$ pulses and normalized amplitude spectra are shown in Figure 3. The black solid line is a $\mathrm{THz}$ reference pulse passing through the empty cell of PP window. The sample pulses transmitted though the same cell filled with the liquid insulating oils were obtained in the time domain. The $\mathrm{THz}$ sample pulses of transmitted or reflected are reduced of amplitude, and delayed of phase compare to passing through the free space. In general, strong reducing of amplitude indicates strong absorption, and large delay of phase indicates large refractive index. The normalized amplitude spectra of insulating oils, which are obtained by Fast Fourier Transform (FFT) of the measured THz pulses, are shown in Figure 3 (b).It shows that reducing of amplitude is attributed to increase of absorption due to aging effect.
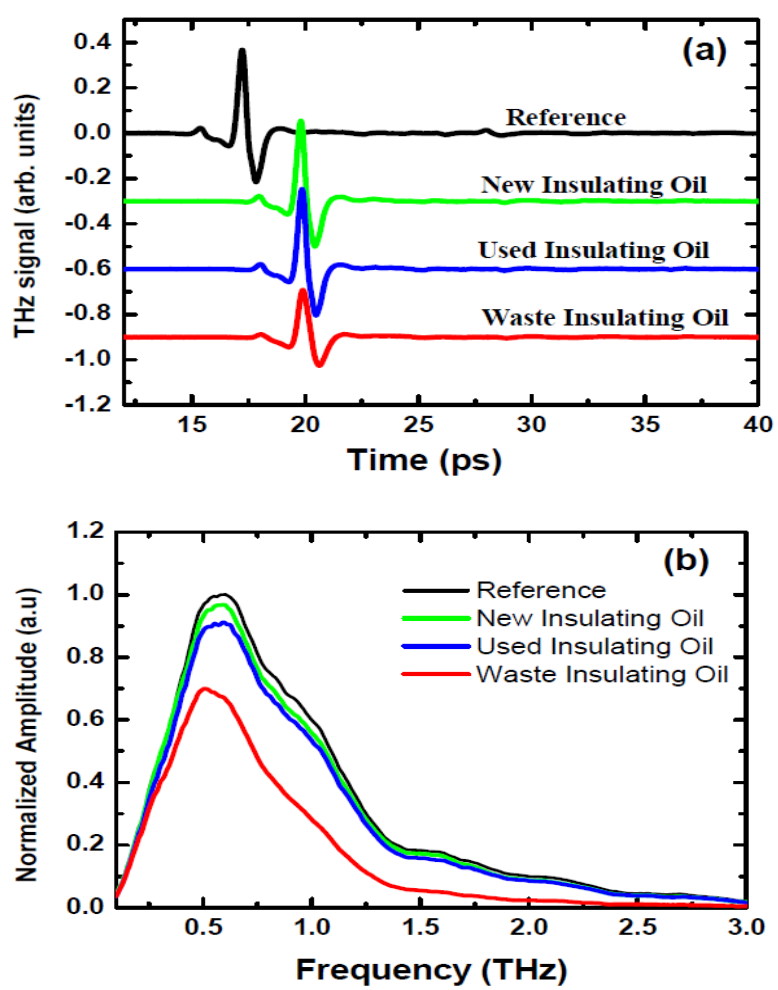

Fig. 3: (A) Measured Thz Pulses. Black Line Is the Reference Pulse Passing Through The Empty Sample Cell. (B) Normalized Amplitude Spectra which are obtained by Fast Fourier Transform (FFT) of the Measured Thz Pulses.

Assuming the normal incidence of $\mathrm{THz}$ pulse and non-scattering surface of sample surface, THz-TDS measurement can describe with the Fresnel equation. The refractive index, absorption coefficient, and dielectric constant of insulating oils can be calculated from measured $\mathrm{THz}$ pulse in time domain and phase components which are obtained from FFT of THz pulse in frequency domain. Figure 4 shows the refractive index and absorption coefficient of insulating oils. The refractive index of 'new insulating oil' and 'used insulating oil' exhibit constant values of 1.455 and 1.463 in the full frequency range. But the refractive index of "waste insulating oil' exhibits various values of $1.466 \sim 1.485$ with the frequency, which is relatively high. It shows that high refractive index and changing of refractive index value with the frequency are attributed to aging effect of insulating oils. The absorption coefficient of 'new insulating oil' and 'used insulating oil' exhibit constant values of 0 $\sim 1 \mathrm{~cm}^{-1}$ and $0.8 \sim 1.8 \mathrm{~cm}^{-1}$ in the full frequency range. But the absorption coefficient of 'waste insulating oil' is changed $1 \sim 17$ $\mathrm{cm}^{-1}$ with the increase linearly of frequency. It is also aging effect of insulating oils.

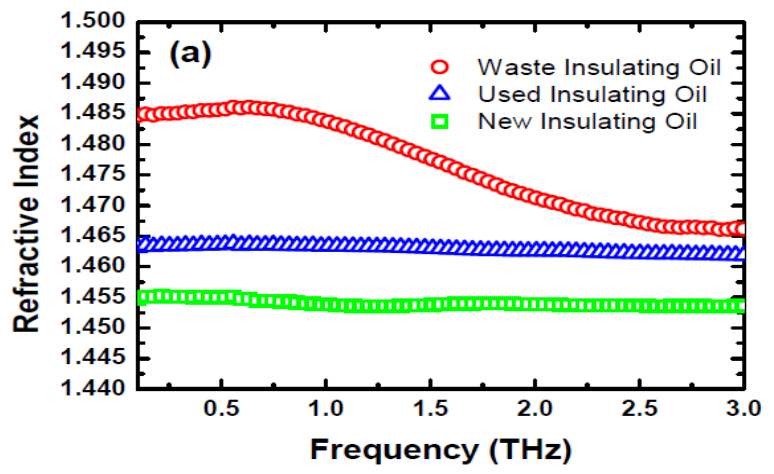




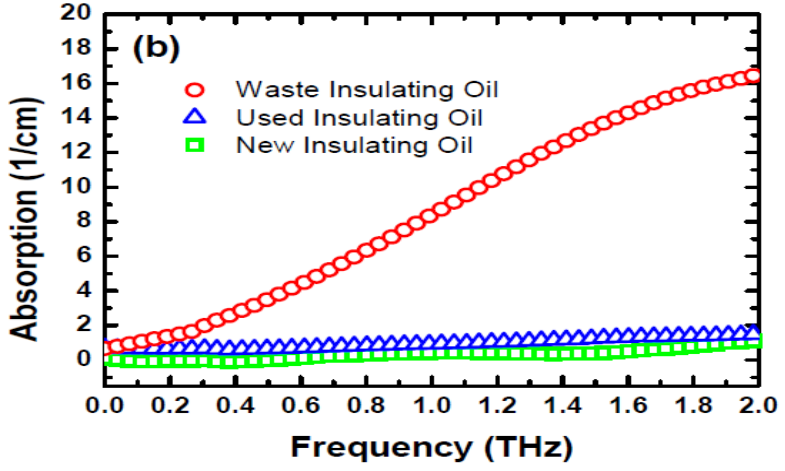

Fig. 4: The Thz Characteristics of Insulating Oils. (A) Refractive Index, and (B) Absorption Coefficient.

The complex dielectric constant $\left(\epsilon(\omega)=\left(n_{r}+n_{i}\right)^{2}\right)$ of insulating oils can be calculated from refractive index and absorption coeffi$\operatorname{cient}\left(\alpha=2 n_{i} \omega / c\right)$ of insulating oils. The real part and imaginary part of dielectric constant are calculated by $\epsilon_{r}=n_{r}^{2}-n_{i}^{2}, \epsilon_{i}=$ $\alpha n_{r} c / \omega$. It is shown in Figure 5. The real dielectric constant of 'new insulating oil' and 'used insulating oil' exhibit constant values of 2.12 and 2.14. But real dielectric constant of 'waste insulating oil' exhibits relative high of $2.15 \sim 2.21$. It is also aging effect of insulating oils.

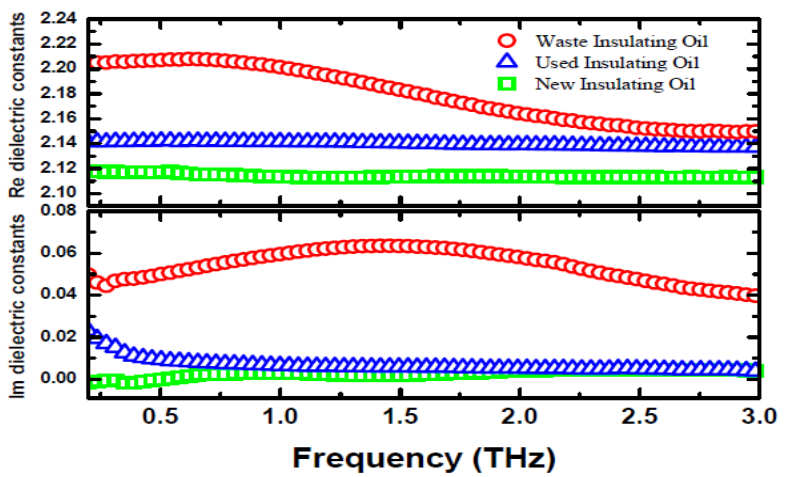

Fig. 5: Dielectric Constants of Insulating Oils Characterized as Functions of Thz Frequency.

The loss coefficient tan $\delta$ of power loss can be calculated from $\tan \delta=\epsilon_{i}(\omega) / \epsilon_{r}(\omega)$. The loss coefficient is shown in Figure 6 The loss coefficient of 'new insulating oil' is nearly zero, which means zero power loss. But the loss coefficient of 'waste insulating oil' exhibits relatively highof $2.0 \sim 3.0$.

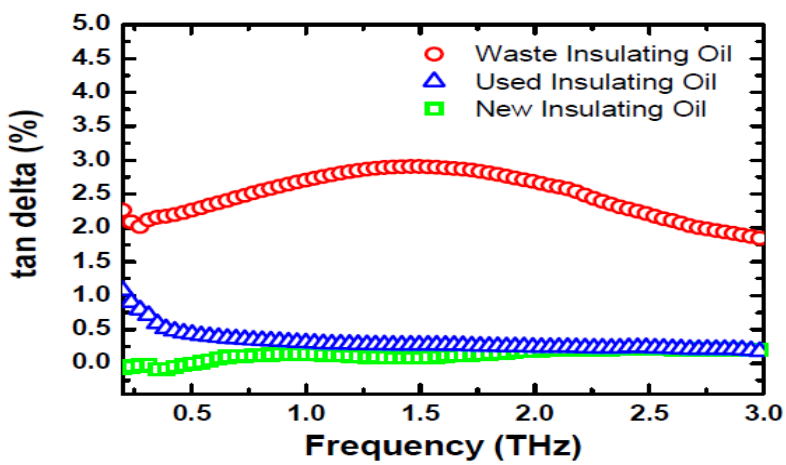

Fig. 6: The Tan Delta of Insulating Oils, which are Indicated Dielectric Power Loss Obtained from Complex Dielectric Constants of Insulating Oils.

\section{Conclusion}

The aging effect of insulating oils of high voltage transformer is characterized by the portable THz-TDS system. The absorption coefficient and power loss coefficient of 'new oil and normal operation oil' exhibit low constant value in the range of $0.1 \sim 3.0 \mathrm{THz}$ frequency. But absorption coefficient of 'waste oil of malfunction' exhibits linearly increase with the increase of frequency. The power loss coefficient of 'waste oil' exhibit relatively high. The portable THz-TDS system is a compact and beam alignment free system. Thus the portable THz-TDS system can be applied to in situ monitoring of transformer in substation. The in situ monitoring of transformer in substation is key play of stable electrical power supply with the low cost.

\section{References}

[1] Husain Z, Malik H, Arif Khan M, Recent Trends in Power Transformer Fault Diagnosis and Condition Assessment, Bulletin of Electrical Engineering and Informatics, 2013, 2(2), pp. 95-104

[2] Prevost T A, Oommen T V, Cellulose insulation in oil-filled power transformers: Part I-History and development, IEEE Electrical Insulation Magazine, 2006, 22(1), pp. 28-35.

[3] Hasmat Malik, AbduiAzeem, Condition assessment of power transformer by swift frequency response analysis, International Journal of Electrical Engineering (IJEE), ISSN 0974-2158, 2011 4(2), pp. 199-207

[4] ChendongX, Monitoring paper insulation aging by measuring furfural content of oil, Proceedings 7th International Symposium High Voltage Engineering, 1991, 26-30: 139-42.

[5] Pukel G J, Muhr H M, Lick W, Transformer diagnostics: Common used and new methods, International Conference on Condition Monitoring and Diagnosis, CMD 2006, Chanwon, Korea, 2006.

[6] Arakelian V G, The long way to the automatic chromatographic analysis of gases dissolved in insulating oil, IEEE Electrical Insulation Magazine, 2004, 20(6), pp. 8-25.

[7] Kan H, Miyamoto T, Makino Y, Namba S, Hara T, Absorption of $\mathrm{CO} 2$ and COgases and furfural in insulating oil into paper insulation in oil-immersed transformers, IEEE International Symposium on Electrical Insulation Conference, 1944, pp. 41-44.

[8] Hosier I L, Vaughan A S, Sutton S J, Davis F J, Chemical and physical properties of aged dodecylbenzene insulating oil, Electrical Insulation and Dielectric Phenomena, 2005, pp. 225-228.

[9] Aka-Ngnui T, Benounis M, Jaffrezic-Renault N, Stevenson I, Beroual A, In situ monitoring of the degradation of insulating oil under AC voltage, Electrical Insulation and Dielectric Phenomena, 2005, pp. 253-256

[10] Jin Y S, Kim G J, Shon C H, Jeon S G, Kim J I, Analysis of petroleum products and their mixtures by using terahertz time domain spectroscopy, Journal of the Korean Physical Society, 2008, 53(4), pp. 1879-1885.

[11] Moller U, Cooke D G, Tanaka K, UhdJepsen P, Terahertz reflection spectroscopy of Debye relaxation in polar liquids, Optical Society of America, 2009, 26(9), pp. A113-A125.

[12] Docherty C J, Johnston M B, Terahertz properties of graphene, Journal of Infrared Milli Terahertz Waves, 2012, 33, pp. 797-815.

[13] Seco-Martorell C, Lopez-Dominguez V, Arauz-Garofalo G, Redo-Sanchez A, Palacios J, Tejade J, Goya's artwork imaging with terahertz waves, Optics Express, 2013, 21(15), pp. 1780017805.

[14] Adam A J L, Planken P C M, Meloni S, Dik J, Terahertz imaging of hidden paint layer on canvas, Optics Express, 17(5), pp. 34073416.

[15] Kang S B, Chung D C, Kim S J, Chung J K, Park S Y, Kim K C, Kwak M H, Terahertz characterization of Y2O3-added AlN ceramics, Applied Surface Science, 388, pp. 741-745.

[16] Markelz A, Whitmire S, Hillebrecht J, Birge R, THz time domain spectroscopy of biomolecular conformation modes, Physics in Medicine and Biology, 2002, 47, pp. 3797-3805. 